\title{
Platelet Inventory Management Program: Development and Practical Experience
}

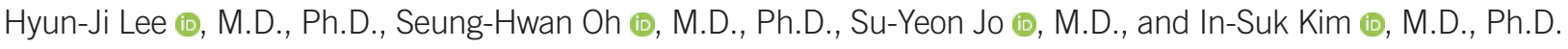 \\ Department of Laboratory Medicine and Research Institute for Convergence of Biomedical Science and Technology, Pusan National University Yangsan \\ Hospital, Yangsan, Korea
}

Background: Patients with ongoing or expected bleeding require platelet (PLT) transfusions; however, owing to the testing required after a blood donation, manufacturing PLT products may take 1.5-2.0 days after a request is made. This supply-demand mismatch leads clinicians to retain spare PLTs for transfusions, leading to increased PLT discard rates. We developed a PLT inventory management program to supply PLTs more efficiently to patients requiring PLT transfusions within the expiration date, while reducing PLT discard rates.

Methods: PLT concentrates (58,863 and 58,357 units) and apheresis products (7,905 and 8,441 units) were analyzed from May 2015 to November 2017 and from December 2017 to January 2020, respectively. We developed a program to manage total PLT inventories and prospective PLT transfusion patients based on blood type, blood product, and remaining period of efficacy; the program facilitates PLT preparation transfer to non-designated patients within the remaining period of efficacy.

Results: The overall PLT concentrate discard rate was 3,254 (2.78\%): 1,811 (3.07\%) units before and 1,443 units $(2.41 \%)$ after program application $(P<0.001)$. The discard rate owing to expiration was reduced from 69 units $(3.81 \%)$ before to two units $(0.14 \%)$ after program application $(P<0.001)$.

Conclusions: This program can guide the allocation of PLT preparations based on the remaining period of efficacy, enabling PLT products to be used before their expiration date and reducing PLT product discard rate.

Key Words: Platelet concentrates, Apheresis, Transfusions, Discard rate, Inventory program, Expiration date
Received: March 9, 2020

Revision received: April 2, 2020

Accepted: August 2, 2020

\section{Corresponding author:}

Hyun-Ji Lee, M.D., Ph.D.

Department of Laboratory Medicine and

Research Institute for Convergence of

Biomedical Science and Technology, Pusan

National University Yangsan Hospital,

20 Geumo-ro, Mulgeum-eup, Yangsan

50612, Korea

Tel: +82-55-360-1875

Fax: $+82-55-360-1880$

E-mail: hilhj1120@gmail.com

\section{INTRODUCTION}

Platelets (PLTs) have a short life span of 10 days; although various methods to increase PLT shelf life in vitro have been investigated, the shelf life of PLTs remains at 5-7 days, depending on the separation method [1, 2]. This leads to a supply-demand mismatch, as it takes 1.5-2.0 days to complete the actual blood screening test after a blood donation [3]. This has led to increased efforts by clinicians to retain more PLTs for transfusions, leading to increased PLT discard rates [1]. Maintaining adequate PLT supply to meet demands is hindered by the short shelf life of PLTs. Additionally, PLT discard rate needs to be reduced to prevent resource waste and patient endangerment owing to PLT deficiency $[4,5]$. Numerous efforts-"up-to-order" rule, automatic dashboard, cooperation with blood supplier, etc.- - have been made to reduce PLT discard rates by as much as $1-5 \%$ [1, 4, 6, 7]; however, PLT expiration continues to be an important cause of PLT discard [5]. 
We have developed a PLT inventory management program that shows the overall status of PLT inventory and allows clinicians to easily change the status of assigned PLTs according to clinical need. The objective of developing this PLT inventory program was to supply PLTs to patients requiring transfusions within the PLT expiration date, while also reducing PLT discard and waste.

\section{MATERIALS AND METHODS}

\section{Evaluation}

The PLT Inventory Management Program was initiated on December 1, 2017 in the Department of Laboratory Medicine and Biomedical Research Institute Pusan National University Yangsan Hospital, Yangsan, Korea. PLT concentrates (58,863 and 58,357 units) and PLT apheresis units (7,905 and 8,441 units) supplied from the Korean Red Cross were evaluated from May 2015 to November 2017 and from December 2017 to January 2020, respectively. The reasons for PLT product discard were classified into improvement of patient condition, deterioration of patient condition, expiration, blood bag problems, and others. Patients aged <one month were classified as neonates; >one month, as children; and $>18$ years, as adults. The time remaining until PLT expiration was defined as time till outdating (TTO). This retrospective study was approved by the Institutional Review Board at Pusan National University Yangsan Hospital, Yangsan, Korea (No. 05-2020-051). This study required neither studyintended blood sampling nor additional interventions; therefore, the requirement to obtain written informed consent from the patients was waived.

\section{Routine process}

In our hospital, it is a standard operating procedure to transfuse ABO Rh-identical PLTs. In transplant patients, minor-incompatible PLTs can be transfused following the approval of a transfusion medicine physician. PLT inventory is usually conducted in the following manner. Depending on the patient's condition, the clinician orders the number of PLT products needed, and the hospital blood bank staff orders the required PLT products from the supplier (e.g., the Korean Red Cross). When the PLT product is received at the hospital, the clinician is notified, and the product is assigned to a patient for transfusion. If the PLT age in the assigned PLT product is approximately four days and the expiration date is imminent, hospital blood bank staff will check with the clinician whether the patient should be transfused. If the clinician decides against the transfusion, then the PLT prod- uct can be transferred to another patient who needs a PLT transfusion but has not yet been assigned a PLT product.

\section{PLT inventory management program design}

As shown in Fig. 1, the PLT inventory management program screen provides information on the status of the patient. Fig. 1 (A) shows the PLT prescription status. In the table listing the expiration date of all PLT products, the number in the box next to the time indicates the expiration date of the available PLT products according to product and blood type. A value of 24 indicates that 24 hours remain until the expiration date. Clicking the number on this screen will search for patients who have been prescribed a transfusion of a specific PLT product. Fig. 1B shows the entire process of transferring the PLT product to another patient. It can be cancelled individually at (6) by clicking the empty box at the end of the patient name and pressing (7) to cancel the assigned PLT products. PLT products can be exchanged between two patients at (9).

\section{Statistics}

Categorical variables were compared between the groups before and after application of the PLT inventory program using Pearson's $\chi^{2}$ test. Numerical data were expressed as mean \pm standard deviation (SD). Differences were statistically analyzed using the t-test. All statistical tests were performed with IBM SPSS Statistics for Windows version 26 (IBM Corp., Armonk, NY, US). $P<0.05$ was considered statistically significant.

\section{RESULTS}

\section{PLT discard rates}

The overall discard rate of PLT concentrates was 3,254 (2.78\%), i.e., 1,811 (3.07\%) units before program application and 1,443 units $(2.41 \%$ ) after program application (Table $1 ; P<0.001$ ). The PLT apheresis discard rate was one unit $(0.02 \%)$ for the entire period, and it occurred post-program application. The reasons for PLT discard are shown in Table 2. The data for improved and worsened patient conditions were similar before and after program application. Discard owing to PLT concentrate expiration decreased from 69 to 0 units, while discard owing to PLT apheresis expiration increased from 0 to two units. The total discard rate owing to expiration was reduced from 69 units (3.81\%) before program application to two units (0.14\%) after program application $(P<0.001)$. When the patients were categorized according to age, the PLT concentrate discard rate was significantly reduced in the neonate and adult groups (15.76\% vs $8.13 \%, P<$ 
Lee $\mathrm{HJ}$, et al.

Platelet inventory management program

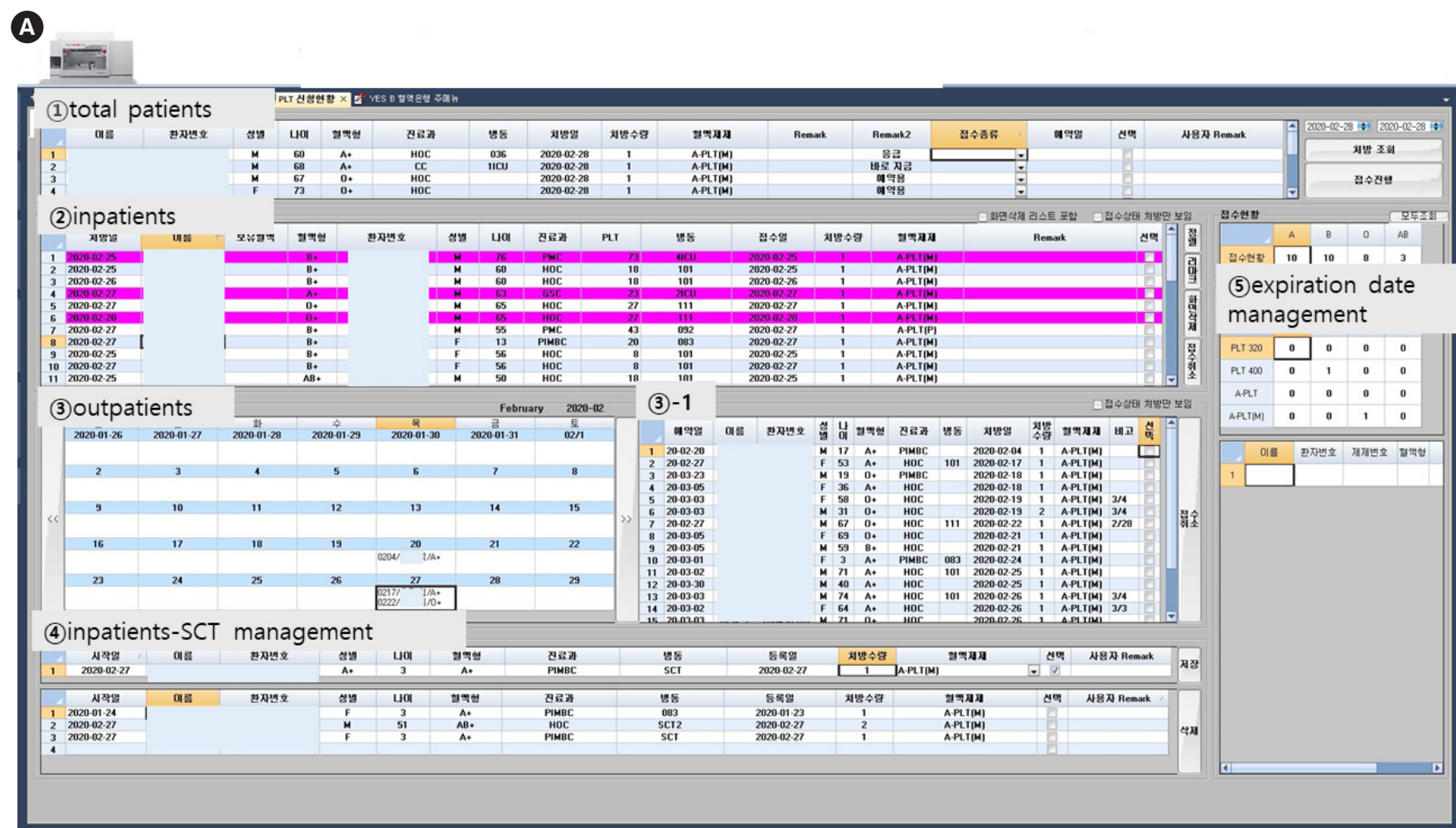

\section{B}

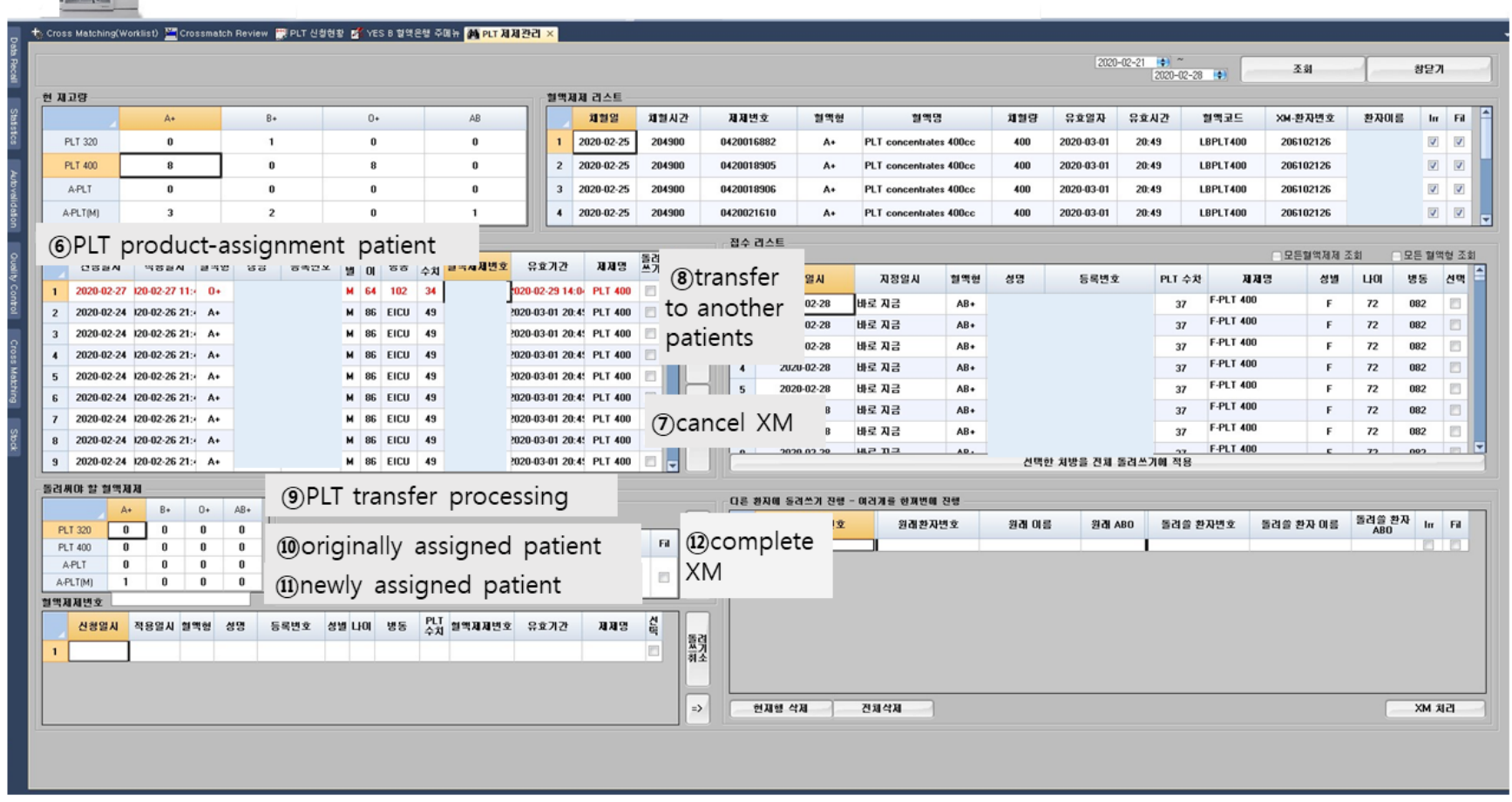

Fig. 1. Screenshot of PLT inventory management program showing $(A)$ the details of the inventory status, $(B)$ the management program for transferring PLT products, and (C) details of each step. 
$0.001 ; 18.35 \%$ vs $20.42 \%, P=0.119 ; 2.26 \%$ vs $1.89 \%, P<0.001$, respectively).

\section{PLT age}

The time interval from blood donation to sample arrival at the hospital blood bank significantly reduced after program application. The mean supply time for PLT concentrates and apheresis products was approximately 8 hours and 2 hours, respectively (Table 3). The age of PLT concentrates at transfusion differed significantly before and after program application (Fig. 2). Age at transfusion of total PLTs was not significant. The TTO of transfused PLT products showed no significant difference in patients with hematologic disease before and after program application

C

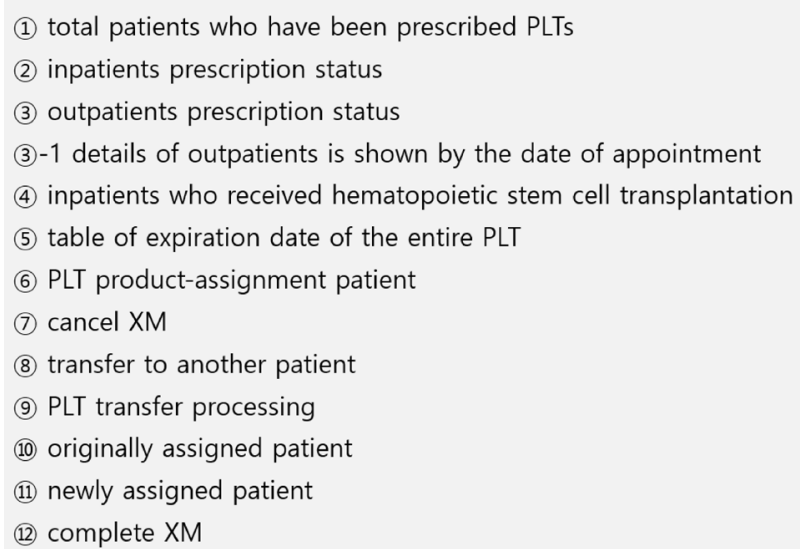

Fig. 1. Continued.

Table 1. Numbers and rate of discarded PLT products (units)

\begin{tabular}{lcc}
\hline Time period & PLT concentrate & PLT apheresis \\
\hline May 2015-November 2017 & $1,811 / 58,863(3.07 \%)$ & $0 / 7,905(0.00 \%)$ \\
December 2017-January 2020 & $1,443 / 58,357(2.41 \%)$ & $1 / 8,441(0.01 \%)$ \\
$P$ & $<0.001$ & 0.516
\end{tabular}

Abbreviation: PLT, platelet.
(Table 4). In other patients, the PLT transfusion rate increased within four hours of TTO after program application $(P<0.001)$.

Table 3. Comparison of PLT age at reception from supplier according to PLT release process before and after PLT inventory management program application

\begin{tabular}{lccc}
\hline PLT type & Group (units) & PLT age (hr) & $P$ \\
\hline Total & Before (66,765) & $43.09 \pm 16.58$ & $<0.001$ \\
& After $(66,795)$ & $35.51 \pm 14.15$ & \\
PLT concentrate & Before $(58,863)$ & $44.55 \pm 16.64$ & $<0.001$ \\
& After $(58,357)$ & $36.24 \pm 14.46$ & \\
PLT apheresis & Before (7,905) & $32.22 \pm 11.33$ & $<0.001$ \\
& After (8,411) & $30.43 \pm 10.41$ & \\
\hline
\end{tabular}

Abbreviation: PLT, platelet.

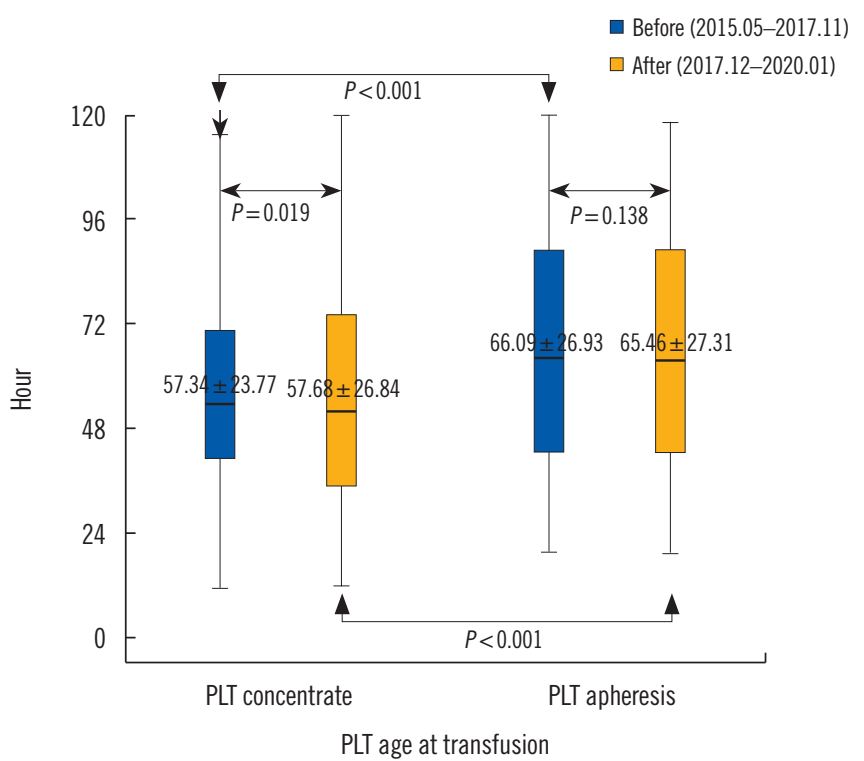

Fig. 2. Comparison of PLT age at transfusion according to type of PLT products before and after PLT inventory program application. Abbreviation: PLT, platelet.

Table 2. Discard rate of PLT products

\begin{tabular}{|c|c|c|c|c|c|c|}
\hline Time period & $\begin{array}{c}\text { Improvement of } \\
\text { patient condition (\%) }\end{array}$ & $\begin{array}{l}\text { Aggravation of patient } \\
\text { condition (\%) }\end{array}$ & $\begin{array}{l}\text { Outdating } \\
(\%)\end{array}$ & $\begin{array}{l}\text { Others* } \\
(\%)\end{array}$ & $\begin{array}{l}\text { Blood bag } \\
\text { defects (\%) }\end{array}$ & Total \\
\hline May 2015-November 2017 & $1,368(75.54)$ & $251(13.86)$ & $69(3.81)$ & $115(6.35)$ & $8(0.44)$ & 1,811 \\
\hline December 2017-January 2020 & $1,192(82.55)$ & $181(12.53)$ & $2(0.14)$ & $63(4.36)$ & $6(0.42)$ & 1,444 \\
\hline May 2015-January 2020 & $2,524(78.54)$ & 431 (13.24) & $71(2.18)$ & $178(5.47)$ & $14(0.43)$ & 3,255 \\
\hline
\end{tabular}

${ }^{*}$ Others include treatment plan changes, poor blood storage conditions with respect to temperature, surgery cancelation, error in blood inventory checks, patient transfers, and absence of patient consent.

Abbreviation: PLT, platelet. 
Table 4. Comparison of time till outdating (TTO) at the time of transfusion

\begin{tabular}{llllcc}
\hline \multirow{2}{*}{ Patients } & & Total & \multicolumn{3}{c}{ Time till outdating } \\
\cline { 4 - 6 } & & (units) & $\geq 4 \mathrm{hr}$ & $<4 \mathrm{hr}$ & $P$ \\
\hline Non- & Before & 44,013 & $43,543(98.95 \%)$ & $470(1.1 \%)$ & $<0.001$ \\
hematology & After & 56,252 & $39,292(69.8 \%)$ & $16,960(30.2 \%)$ & \\
Hematology & Before & 14,847 & $14,636(98.6 \%)$ & $209(1.4 \%)$ & 0.084 \\
& After & 18,451 & $18,148(98.4 \%)$ & $303(1.6 \%)$ & \\
\hline
\end{tabular}

Abbreviation: PLT, platelet.

\section{DISCUSSION}

PLTs are transfused in patients with ongoing or expected bleeding [8]; thus, it is difficult to predict and prepare for a transfusion emergency, and there is a high possibility of patient endangerment if the PLT is not transfused when required [4, 9]. A PLT inventory management program was developed to maintain a good balance between PLT supply and demand. Application of the program reduced discard rates due to expiration.

PLT discard rates vary according to the characteristics of the medical institution as well as patient condition. Studies examining blood product discard rates in 17 European countries reported that the average PLT discard rate was $13 \%$ but varied from $6.7 \%$ to $25 \%$ across institutions $[1,3,6,10]$. International reports examining the reasons for PLT discard suggest that European countries and the United States have a higher expiration rate [1]. Survey results from the American Association of Blood Banks revealed that $11.5 \%$ of apheresis PLTs had expired and $10.5 \%$ of hospitals postponed regular surgery owing to a lack of PLT supply [11]. Despite the high discard rate, there was a lack of PLT preparations, leading to surgeries being canceled; this demonstrated the difficulty in maintaining adequate PLT supply to meet demands. A review from a single center in Korea examining the reasons for blood product discard reported that the PLT discard rate is approximately $0.3 \%$, and the most common reason for discard was expiration [12]. The overall PLT discard rate at our hospital was $2.44 \%$ on an average for a period of 57 months. Before the program was applied, 69 expired PLT products were identified $(6.34 \%$ of discarded PLT products during the same period), while only two expired PLT products $(0.14 \%$ of discarded PLT products during the same period) were discarded after the inventory program was applied.

Various methods have been applied worldwide to reduce PLT discard rates $[4,6,7]$; of these, the "order-up-to" rule is known to be the most useful. This rule indicates that the target PLT in- ventory has to be maintained and if the reserve is lower than the target inventory, sufficient units need to be procured to maintain the target inventory [4]. One hospital that applied the "order-upto" rule conducted a theoretical simulation and practical analysis to reduce PLT discard rates and increase the use of younger PLTs during transfusion. For this, a slightly higher number of PLT units was prepared during holidays according to PLT orders. After five years of using the "order-up-to" program, the expiration rate of PLT products decreased from 5.2\% to $1.3 \%$ and PLT age at transfusion decreased from a mean of 3.74 to 3.27 days [3].

Numerous hospitals have applied interventions to change the ordering system, apply the "order-up-to" rule, inform daily PLT discard status, and facilitate exchanges between local hospitals to reduce PLT discard rates owing to expiration. As a result, PLT discard due to expiration has decreased, depending on the hospitals [5]. In hospitals that use the "order-up-to" rule and the "automatic dashboard" (a program that monitors the hospital's PLT inventory and patients' PLT orders), the PLT discard rate decreased after using the dashboard, and the mean PLT age at transfusion decreased from 3.60 days before dashboard use to 3.46 days after dashboard use (approximately 3.4 hours) [6]. One hospital reported that PLT transfusion indications, in-hospital inventory management, and close coordination with the PLT product supplier kept the discard rate of PLT apheresis products $<1 \%$ [7]. The application of other interventions, such as a decrease in PLT age at transfusion, together with the "order-up-to" rule, may lead to more effective PLT transfusions.

In our hospital, there was no decrease in PLT age at transfusion. However, the discard rate remains higher than that of other hospitals because the "order-up-to" rule has not been applied. As each hospital has its own discard rate (generally $<1 \%$ ) [12$14]$, it is important to address the hospital-specific reasons underlying blood product discard to reduce waste $[15,16]$. For example, the PLT discard rate was reduced in hospitals where efforts were made to correct changes in blood bag temperature or the return time during PLT transfer [15], as well as educate transfusion staff regarding blood donations [16]. Our hospital's high PLT discard rate is likely related to PLT products being preassigned to patients requiring transfusions, without using the "order-up-to" rule. This strategy was implemented when our hospital opened 12 years ago and was only treating a few patients for PLT transfusions. Our hospital has grown and now treats a larger number of patients requiring transfusions. Therefore, we plan to change the PLT management guidelines to administer PLT products based on their TTOs, as well as applying an inven- 
tory management policy based on the "order-up-to" rule, which matches the ordered PLT products to the number of in-stock PLT preparations. In addition, educating clinicians regarding these policies and our PLT management program may help reduce the PLT discard rate and PLT age at transfusion.

Our study has several limitations. First, it is limited by its retrospective, single center design. Second, it did not consider changes in the disease group of PLT transfusion patients during the total evaluation period.

In conclusion, we have described our experience in developing a program that can deliver PLT preparations based on their remaining TTO to help ensure that these preparations are used before their expiration date and subsequently reduce the discard rate. Maintaining our efforts to reduce PLT discard using the PLT inventory management program will lead to more efficient PLT transfusions.

\section{ACKNOWLEDGEMENTS}

The authors wish to thank Cho, Hang-Pyo from Ortho Clinical Diagnostics for implementing the PLT inventory management program.

\section{AUTHOR CONTRIBUTIONS}

HJL and ISK designed the study; HJL analyzed the data and wrote the manuscript; and SHO and SYJ edited the manuscript. All authors reviewed and approved the manuscript.

\section{CONFLICTS OF INTEREST}

No potential conflicts of interest relevant to this paper were reported.

\section{RESEARCH FUNDING}

This work was supported by the Research Institute for Convergence Biomedical Science and Technology (30-2019-008), Pusan National University Yangsan Hospital.

\section{ORCID}

Hyun-Ji Lee

https://orcid.org/0000-0002-9021-5632
Seung-Hwan Oh

https://orcid.org/0000-0002-1946-9939

Su-Yeon Jo

In-Suk Kim https://orcid.org/0000-0001-7735-2897 https://orcid.org/0000-0002-7243-9173

\section{REFERENCES}

1. Veihola M, Aroviita P, Linna M, Sintonen $H$, Kekomäki R. Variation of platelet production and discard rates in 17 blood centers representing 10 European countries from 2000 to 2002. Transfusion 2006;46:991-5.

2. Handigund M and Cho YG. Insights into platelet storage and the need for multiple approaches. Ann Clin Lab Sci 2015;45:713-9.

3. de Kort W, Janssen M, Kortbeek N, Jansen N, van der Wal J, van Dijk N. Platelet pool inventory management: theory meets practice. Transfusion 2011;51:2295-303

4. Blake JT. On the use of operational research for managing platelet inventory and ordering. Transfusion 2009;49:396-401.

5. Flint AW, McQuilten ZK, Irwin G, Rushford K, Haysom HE, Wood EM. Is platelet expiring out of date? A systematic review. Transfus Med Rev 2020;34:42-50.

6. Gomez AT, Quinn JG, Doiron DJ, Watson S, Crocker BD, Cheng CK. Implementation of a novel real-time platelet inventory management system at a multi-site transfusion service. Transfusion 2015;55:2070-5.

7. Fuller AK, Uglik KM, Braine HG, King KE. A comprehensive program to minimize platelet outdating. Transfusion 2011;51:1469-76.

8. Fillet AM, Desmarets M, Assari S, Quaranta JF, François A, Pugin A, et al. Blood products use in France: a nationwide cross-sectional survey. Transfusion 2016;56:3033-41.

9. Fedele PL, Polizzotto MN, Grigoriadis G, Waters N, Comande M, Borosak $\mathrm{M}$, et al. Profiling clinical platelet and plasma use to inform blood supply and contingency planning: PUPPY, the prospective utilization of platelets and plasma study. Transfusion 2016;56:2455-65.

10. van Dijk N, Haijema R, van der Wal J, Sibinga CS. Blood platelet production: a novel approach for practical optimization. Transfusion 2009; 49:411-20.

11. Whitaker B, Rajbhandary S, Kleinman S, Harris A, Kamani N. Trends in United States blood collection and transfusion: results from the 2013 AABB Blood Collection, Utilization, and Patient Blood Management Survey. Transfusion 2016;56:2173-83.

12. Jo SA, Hwang WJ, Kim SY, Kim YJ, Kim HH, Chang CH, et al. Study for efficient blood management using evaluation the blood wastage statements in Pusan University Hospital. Korean J Blood Transfus 2008;19: 25-32.

13. Kim BC, Seo YI, Chai DR, Shin JW, Choi TY. Analysis of discarded blood components at a university hospital in Korea. Korean J Blood Transfus 2011;22:120-6.

14. Kwon JR, Lee SW, Cho YH, Kwon YH, Kim HJ, Lee HJ, et al. An analysis of blood usage and blood wastage in Korea hospitals in 2008. Korean J Blood Transfus 2009;20:184-94.

15. Collins RA, Wisniewski MK, Waters JH, Triulzi DJ, Yazer MH. Effectiveness of multiple initiatives to reduce blood component wastage. Am J Clin Pathol 2015;143:329-35.

16. Javadzadeh Shahshahani $\mathrm{H}$ and Taghvai N. Blood wastage management in a regional blood transfusion centre. Transfus Med 2017;27(S5):34853. 